\title{
Modeling of Non-isothermal Cryogenic Fluid Sloshing
}

\author{
Juan H. Agui* and Jeffrey P. Moder ${ }^{\dagger}$ \\ NASA Glenn Research Center, Cleveland, OH, 44135
}

\begin{abstract}
A computational fluid dynamic model was used to simulate the thermal destratification in an upright self-pressurized cryostat approximately half-filled with liquid nitrogen and subjected to forced sinusoidal lateral shaking. A full three-dimensional computational grid was used to model the tank dynamics, fluid flow and thermodynamics using the ANSYS Fluent code. A non-inertial grid was used which required the addition of momentum and energy source terms to account for the inertial forces, energy transfer and wall reaction forces produced by the shaken tank. The kinetics-based Schrage mass transfer model provided the interfacial mass transfer due to evaporation and condensation at the sloshing interface. The dynamic behavior of the sloshing interface, its amplitude and transition to different wave modes, provided insight into the fluid process at the interface. The tank pressure evolution and temperature profiles compared relatively well with the shaken cryostat experimental test data provided by the Centre National D'Etudes Spatiales.
\end{abstract}

\section{Nomenclature}

$=$ acceleration

$=$ energy

$=$ surface force

$=$ thermal conductivity

$=$ mass flow rate

$=$ pressure

$=$ source term

$=$ time

$=$ Temperature

$=$ velocity

$=$ lateral displacement

$=$ displacement amplitude of oscillation

\section{Greek}

$\alpha \quad=$ Volume of Fluid fraction

$\gamma \quad=$ curvature

$\mu \quad=$ effective viscosity

$\rho \quad=$ density

$\sigma=$ accommodation coefficient

$\tau=\quad=$ time constant (ramp up of shaking)

$\omega=$ shaking frequency

Subscripts

eff = effective

$i \quad=$ interface

$v \quad=$ saturated vapor

\footnotetext{
* Aerospace Engineer, Fluid Physics and Transport Branch, 21000 Brookpark Rd, Mail Stop 77-5, AIAA Member.

${ }^{\dagger}$ Aerospace Engineer, Combustion Branch, 21000 Brookpark Rd, Mail Stop 5-10, AIAA Member.
}

American Institute of Aeronautics and Astronautics 


\section{Introduction}

$\mathrm{F}$ uel or propellant sloshing in launch vehicles can have challenging and detrimental effects on the performance of the vehicle. Any sudden propulsive maneuvers can instantly induce large motion sloshing behavior. Due to the large proportion of propellant mass these vehicles carry, the sloshing of the liquid fuel against the tank walls and inertial response of the liquid mass can produce large or critical dynamic loads on the vehicle. In addition, the liquid and vapor phase can undergo significant thermodynamic changes that can also produce strong pressure waves and variations. Therefore understanding the real-time dynamics and thermodynamics of cryogenic tank sloshing is vital to predicting and controlling the tank temperature and pressure response on launch vehicles, or future propellant depots.

A partially filled tank is most susceptible to sloshing when undergoing lateral vibrational or oscillatory motion. The ullage region in cryogenic propellant tanks will typically have significant temperature variation between the tank walls and liquid/ullage interface (thermal stratification). Motion of the liquid/ullage interface of sufficient amplitude during sloshing events can lead to thermal destratification and a significant decrease in tank pressure. The dynamics of the sloshing phenomenon has been studied quite extensively since the 1960's (see e.g. Refs 1 and 2). Analytical studies of sloshing in cylindrical tanks have also been conducted ${ }^{3}$. However the interest in thermal destratification is more recent. Thermal de-stratification and pressure reduction from lateral sloshing has been investigated experimentally by Lacapere et al. ${ }^{4}$, Arndt et al. ${ }^{5}$ and Himeno et al ${ }^{6}$.

This paper will describe the full three-dimensional CFD simulation and comparison with experimental data of an upright $182 \mathrm{~mm}$ diameter and $800 \mathrm{~mm}$ tall cylindrical tank approximately half filled with liquid nitrogen and subjected to forced sinusoidal horizontal shaking. The liquid nitrogen sloshing experiments ${ }^{4}$ were performed in 2002 by the Advanced Technical Division of Air Liquid as part of the French-German COMPERE (COMPortement des ERgols dans les REservoirs) program. Information on the experiment was provided to NASA by Centre National D'Etudes Spatiales (CNES) of France under an Implementing Arrangement on CFD benchmarking for cryogenic propellant tank analysis. Improving the capability of computational tools to predict fluid dynamic and thermodynamic behavior in cryogenic propellant tanks under settled and unsettled condition is part of the work supported under the previous NASA Cryogenic Propellant Storage and Transfer project and under the current NASA Evolvable Cryogenics project.

\section{Computational Model}

The computational (CFD) model solves for the equations of continuity, momentum, and energy. The Volume of Fluid (VOF) method is employed to track the liquid and gas phases and their interface in the computational domain. The energy and temperature are defined as mass average scalars, e.g.:

$$
E=\frac{\sum_{q=1}^{2} \alpha_{q} \rho_{q} E_{q}}{\sum_{q=1}^{2} \alpha_{q} \rho_{q}}
$$

Here $\alpha, \rho, \mathrm{E}$ are the phase fraction, density, and energy respectively. The phase properties in a two-phase system are determined by their volume-fraction averages quantities for density, effective viscosity and thermal conductivity as:

$$
\rho=\sum_{q=1}^{2} \alpha_{q} \rho_{q}, \quad \mu_{e f f}=\sum_{q=1}^{2} \alpha_{q} \mu_{e f f} q, \quad k_{e f f}=\sum_{q=1}^{2} \alpha_{q} k_{e f f} q
$$

The mass conservation of the VOF phase is provided by

$$
\frac{1}{\rho_{q}}\left[\frac{\partial}{\partial t}\left(\alpha_{q} \rho_{q}\right)+\nabla \cdot\left(\alpha_{q} \rho_{q} \vec{v}_{q}\right)=S_{\alpha_{q}}\right]
$$

where $S$ is a source term to account for mass generation and sink sources.

A Continuum Surface Force (CSF) model proposed by Brackbill et al. ${ }^{7}$ was implemented to incorporate the effects of surface tension attributed to the VOF phases, and defined by:

American Institute of Aeronautics and Astronautics 


$$
F_{v o l}=\sum_{\text {pairs } i j, i<j} \sigma_{i j} \frac{\alpha_{i} \rho_{i} \gamma_{j} \nabla \alpha_{j}+\alpha_{j} \rho_{j} \gamma_{i} \nabla \alpha_{i}}{\frac{1}{2}\left(\rho_{i}+\rho_{j}\right)}
$$

where $\gamma=\nabla \cdot \hat{n}$ is the curvature on each of the surfaces $(i)$ on the cell and $\hat{n}$ is the unit normal vector of the surface.

The ullage was represented by a compressible ideal gas. Simulations were performed treating the flow as laminar or turbulent. For turbulent simulations, the $\kappa-\omega$ SST turbulence model of Menter et al. ${ }^{8}$ with a variable turbulent damping value of 10 was used. A laminar simulation was also performed for comparison. The thermodynamic properties of the phases was kept constant during the simulation. A second order upwind scheme was used for discretization of the turbulence and momentum equations.

\section{A. Computational Grid}

A full three-dimensional (360 degree) grid was used in order to account for any asymmetric effects, in particular from the interaction of the highly unsteady flow in the two phases, swirling surface waves, and the unsteady sloshing dynamics. The grid consisted of structured hexagonal cells with some skewness of the cells near the cylindrical wall. Three different resolution and grid density configurations were investigated to study their effects on the thermodynamic variables and the flow evolution. Figure 1 shows the different computational grids. Here the cell dimension refers to the height of the cell which was the controlling dimension specified in the gridding process (the arc length of the outer cells on the tank wall were also similarly specified). The other dimensions were automatically determined by the gridding software algorithm and were typically smaller than the controlling dimension. The lowest resolution grid (left), having 579423 cells, was composed of $3 \mathrm{~mm}$ cells in the mid-region of the tank to resolve the sloshing dynamics and thermal destratification in the interface region. The top and bottom portions of this grid, where the flow is expected to be relatively subdued, consisted of $10 \mathrm{~mm}$ cells at the very top and bottom rows of cells respectively and progressively got smaller as the cells approached the mid-region of finer cells. The medium resolution grid (middle), consisting of 741273 cells, was similar to the lowest resolution grid except that the fine $3 \mathrm{~mm}$ cells of the middle region extended to the top wall, covering the top $3 / 4$ of the tank, and the bottom region grid started out with $20 \mathrm{~mm}$ cells on the bottom wall. The highest resolution grid consisting of close to a million cells (984048) had a very narrow fine grid region of $1 \mathrm{~mm}$ cells in the neighborhood of the free interface and transitions to $3 \mathrm{~mm}$ cells on the top and bottom regions.

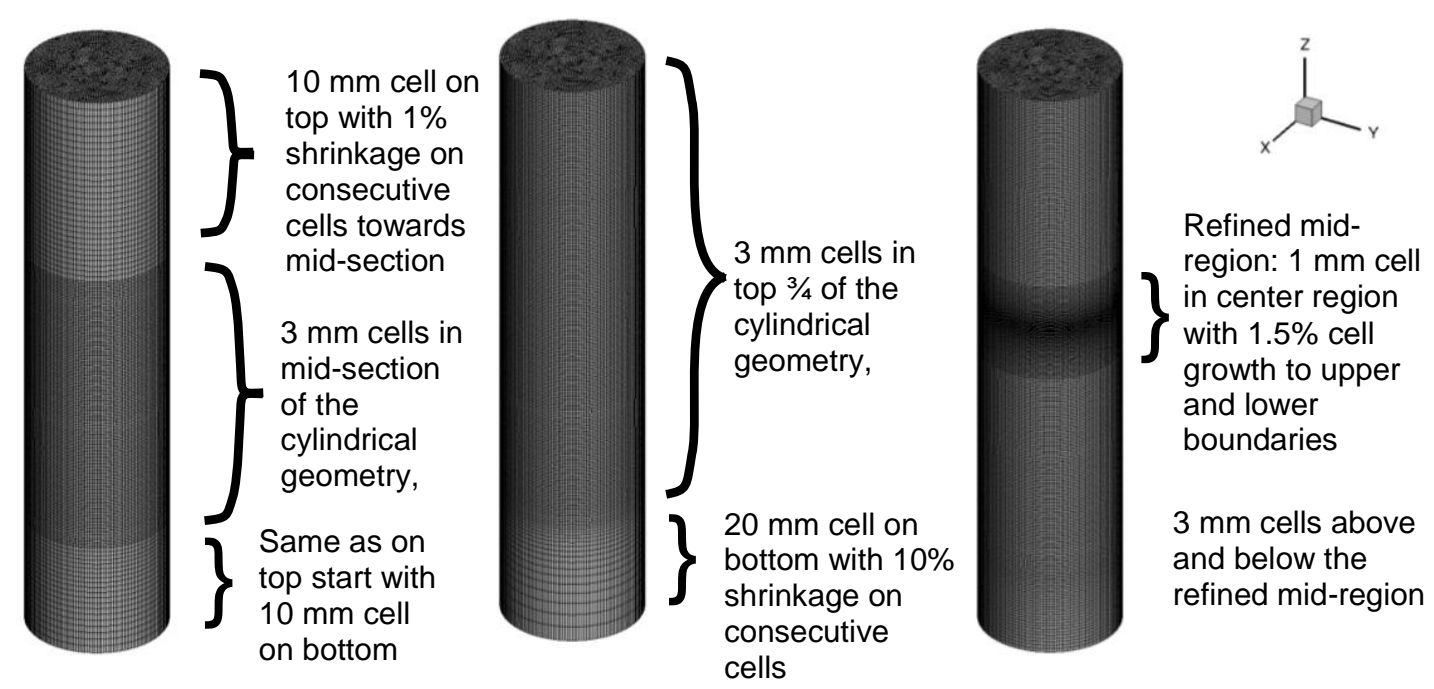

Figure 1: Three computation grids (coarse to very fine resolution from left to right) 


\section{B. Non-inertial grid sloshing model}

The tank was modeled in a non-inertial grid and coordinate system. To account for the lateral shaking of the tank, source terms were added to the momentum and energy equations that simulated the inertial forces, corresponding energy transfer, and reaction forces by the tank. The momentum source term (in the direction of forced lateral shaking) is given by :

$$
S_{x-\text { тот }}=-a \rho
$$

The energy source due to forced lateral shaking is given by:

$$
S_{x-\text { energu }}=-a \rho u=S_{x-m o m} u
$$

The physical lateral shaking motion of the tank was represented by the product of a sinusoidal and a timedependent function. The lateral shaking ramps up from the initial static position to the maximum amplitude within seconds after the shaking is started. Equation 7 gives the displacement in terms of the sinusoidal shaking and the exponential ramp up:

$$
x=X \sin (\omega t)\left[1-e^{\frac{-t}{\tau}}\right]
$$

$X, \omega$, and $\tau$ are the shaking amplitude, frequency, and exponential time constant respectively. Eq. (7) is incorporated into the source terms of Eqs. 5 and 6. The time constant, $\tau$, was calculated from the CNES video data of the shaken cryostat.

\section{Interfacial mass transfer model}

The kinetics-based Schrage ${ }^{9}$ equation is used to model the interfacial mass transfer due to evaporation and condensation. The net mass transfer is given as a mass flux vector between phases:

$$
S_{\alpha_{q}}=\dot{\mathbf{m}}_{i} \cdot \mathbf{A}_{i,}\left[\frac{k g}{m^{3} \cdot \mathrm{sec}}\right]
$$

Here $\dot{m}$ is expressed in terms of Schrage's relation as:

$$
|\mathbf{m}|=\left(\frac{2 \sigma}{2-\sigma}\right)\left(\frac{M}{2 \pi R}\right)^{1 / 2}\left(\frac{P_{i}}{T_{i}^{1 / 2}}-\frac{P_{v}}{T_{v}^{1 / 2}}\right),\left[\frac{k g}{m^{2} \cdot \sec }\right]
$$

where $A_{i}=|\nabla \alpha|, \sigma$ is the accommodation coefficient, and $P$ and $T$ are the pressure and temperature at the saturated vapor state $(v)$ and at the interface (i). This relation incorporates an accommodation coefficient, $\sigma$, which dictates the rate of mass transfer between the phases at conditions near the saturation point. The sign of the accommodation coefficient indicates, depending on convention, whether the liquid is evaporating or condensing.

* Note that source terms are given in terms of the rate of change of the variable of interest. For instance, the source term for momentum will have units of mass/length $/$ time $^{2}$.

American Institute of Aeronautics and Astronautics 


\section{Model Setup}

As starting conditions, the tank is initially self-pressurized and thermally stratified by imposing an initial temperature field provided by the CNES experimental data. The initial thermal stratification is mostly confined to the ullage region of the tank. This initial condition simulates the effects of self-pressurization that a tank may typically undergo prior to launch. The computational domain is initially partitioned (patched) so that it is filled with liquid nitrogen to a height of $450 \mathrm{~mm}$ and gaseous nitrogen above that in the ullage region. The thermodynamic properties of both phases are kept constant at saturation values of the initial pressure $(2.5 \mathrm{kPa})$. In addition, several local heat loads, shown in Fig. 2, are specified along the cylindrical tank wall that remained constant during the simulation. The tank is shaken sinousiodally and laterally at a frequency of $2.1 \mathrm{~Hz}$ and a displacement of $3 \mathrm{~mm}$. The $2.1 \mathrm{~Hz}$ is close to the natural frequency of the partially filled tank system. ${ }^{4}$

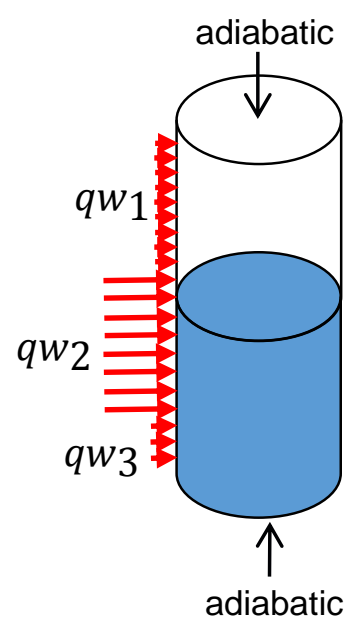

Figure 2: Thermal boundary conditions on the tank.

\section{Results}

\section{A. Pressure evolution}

A set of simulations over the initial shaking period were performed to assess the sensitivity to the effects of the physical models and grid resolutions incorporated in the simulations. Figure 3a shows the effects of the interfacial mass transfer and inertial shaking. The pressure has been normalized by the initial reference pressure. As seen in these plots, the independent contribution from either interfacial mass transfer, i.e. evaporation and condensation, and inertial shaking on the pressure was moderate. However, when both sources are combined the effect on the pressure is significant. In fact, the decrease in pressure in this case is more than the additive effects of the individual contributions, suggesting perhaps a non-linear coupling between these two effects. The effect of grid resolution is presented in Fig. $3 \mathrm{~b}$ which shows a sudden convergence of the higher resolution grids. This seems to indicate that the flow and thermal scales were not well resolved when modeled with the coarse grid (which increases to $10 \mathrm{~mm}$ cells at the top of the tank).

The effects of the rate of mass transfer at the interface, controlled by the accommodation coefficient in the model, was also investigated. The value of the accommodation coefficient produced large effects on the depressurization of the tank at low values. Figure 4 shows a large increase in depressurization between the values of 0.0001 and 0.001 . At values between 0.01 and 0.1 , the effects on the tank depressurization were minor. It is clear that the model is quite sensitive to small changes in the rate of mass transfer at low ranges of mass transfer rates, and insensitive at larger rates.

The modeling data is compared to the CNES experimental data in Fig. 5. The pressure during the initial phase decays more gradually in the experimental case than in the modeling results. Although a time constant (in Eq. 7) based on the experimental data was incorporated into the model, the modeling results could not reproduce the more 
gradual experimental depressurization. Two cases of the modeling data are plotted for comparison. In the case where the fluid flow in the liquid and ullage region where modeled as laminar the overall depressurization is slow compared to the experimental data. When turbulence is modeled, using a $\kappa-\omega$ SST model, the effects on the thermal mixing are significant and produces a larger drop in the pressure. In fact the pressure in the turbulent case approaches the experimental data at the latter stages, approximately after the 20 second mark.

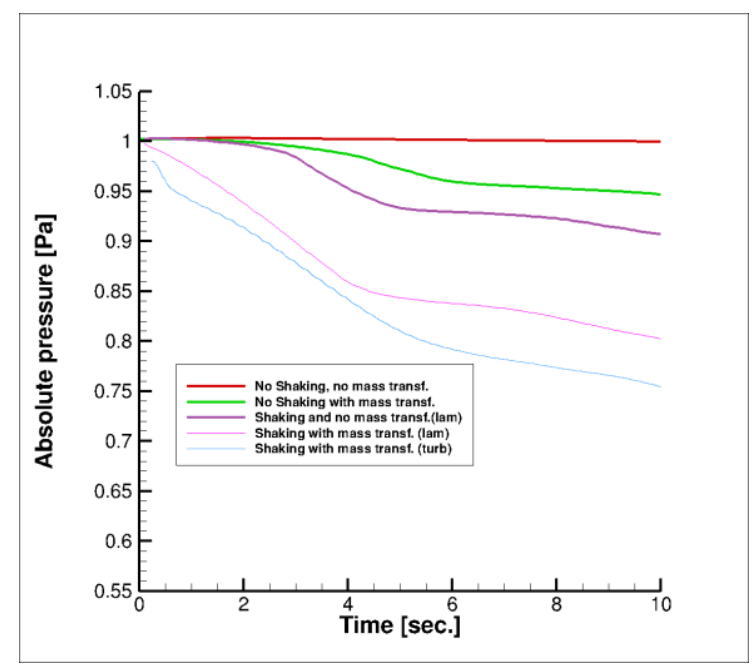

(a)

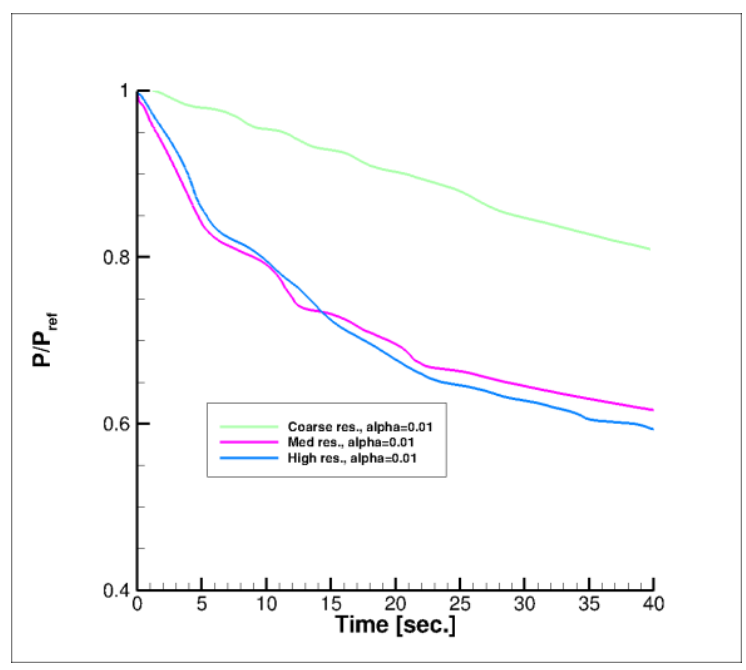

(b)

Figure 3: Pressure plots: (a) comparison different models, (b) comparison of grid resolution.

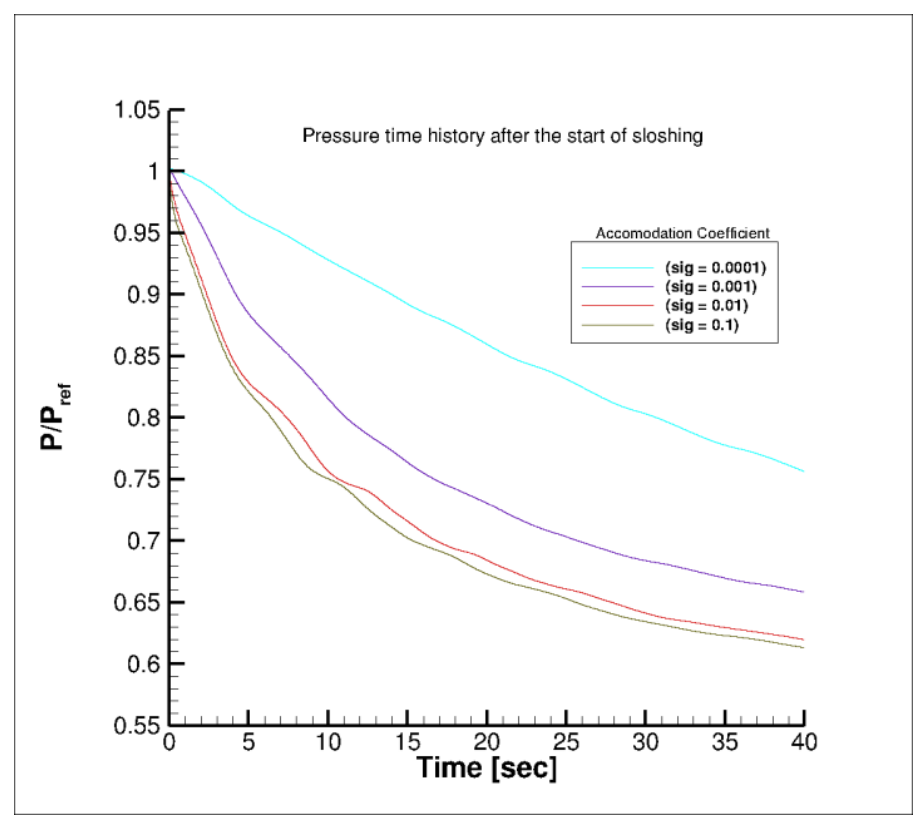

Figure 4: Pressure time history plots for different values of the accommodation coefficient 


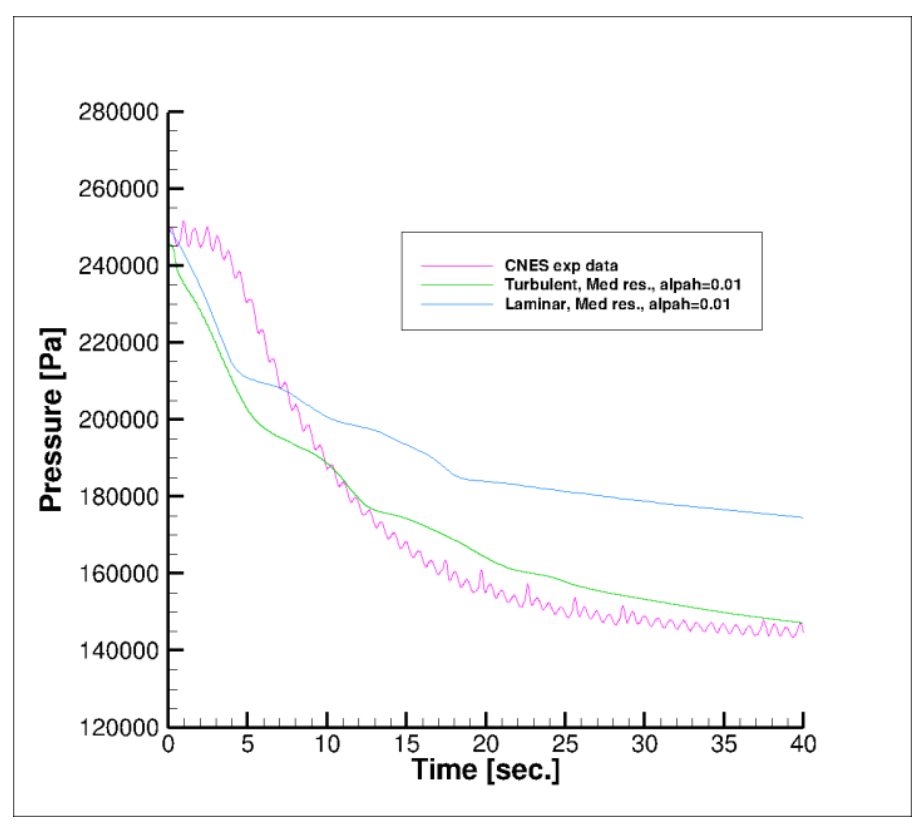

Figure 5: Pressure plot comparing modeling and experimental data.

\section{Sloshing dynamics and de-stratification}

One of the first set of observations obtained from the simulation was on the dynamics of the sloshing free surface wave which seemed to evolve over different stages. This aspect of the sloshing process has implications on the ullage region since the interactions occurring at the interface have a strong bearing on the thermodynamics near the interface and throughout the ullage. Three distinct transitional phases in wave structural dynamics are shown in Fig. 6 . Here the interface is represented by an iso-surface corresponding to a liquid volume of fluid fraction equal to 0.5. The computational results incorporates the interfacial mass transfer (with an accommodation coefficient of 0.01) and inertial shaking models. The first transition occurred at around 4 seconds after the start of shaking. Secondary wave modes were generated along the liquid/ullage interfaces indicated by the folds or creases in the iso-VOF surface. Prior to this the interface was smooth with no creases. Slightly later, at 5 seconds, the interface showed signs of wave breaking at the periphery where pockets of the liquid nitrogen are ejected from the free surface. Then, at 7.8 seconds, a dominant and mainly persistent tangential (swirl) wave mode appears (the grid on the initial free surface is provided to show the tilting or precessing of the free surface in the process of swirling).

$$
\mathrm{t}=4 \mathrm{sec}
$$
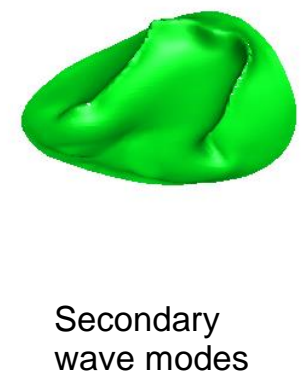

$$
\mathrm{t}=5 \mathrm{sec}
$$

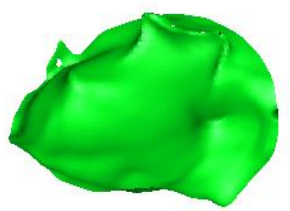

$$
\begin{aligned}
& \text { Wave } \\
& \text { breaking }
\end{aligned}
$$

$\mathrm{t}=7.8 \mathrm{sec}$

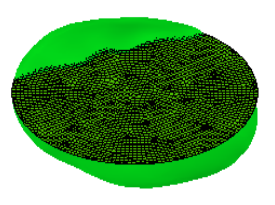

Tangential/Circumferential mode

Figure 6: Sloshing dynamic transitions.

The temperature contour plots in Fig. 7 show the evolution of the thermal de-stratification. The temperature has been normalized by a reference initial liquid nitrogen temperature provided by CNES under the Implementation 
Agreement. Beginning at the 5 second mark, the initially well thermally stratified ullage starts to show a significant convective mixing layer (large scale structures) in the region above the interface. The thermal mixing layer quickly grows and propagates upward into the ullage, reaching the top of the tank at around 25 seconds when temperature variations there are first seen. At 40 seconds the thermal stratification is significantly altered with maximum temperature ratio in the ullage reduced to about $80 \%$ of the initial normalized temperature.

It was also found that the sloshing motion produces different stages of periodicity and intermittency of the temperature field. In Fig. 8a the local temperature history at positions within $2 \mathrm{~cm}$ of the interface (shown as the 0.4 to $0.47 \mathrm{~m}$ vertical position values from the tank bottom) show pronounced large amplitude periodic behavior. This periodic behavior persists for about 10 seconds slightly after the start of shaking, and then transitions suddenly into an equilibrium state characterized by low amplitude random fluctuations. In the central region of the ullage however, at a vertical position of $0.6 \mathrm{~m}$, the temperature is characterized by an initial quiescent period of about 6 seconds followed by one dominated by a very large amplitude intermittency. After this center-ullage temperature transient, the temperature transitions to one exhibiting a lower amplitude fluctuating random behavior. It appears that the thermal convective motion evolves on two distinct spatial and time scales. In the mixing layer above the free surface, the convective thermal transport evolves more rapidly but is confined to a narrow layer, i.e. smaller temporal and spatial scales. By comparison, in the central ullage region the larger spatial convective structures evolve over a corresponding longer time scale.

Figure $8 \mathrm{~b}$ shows the vertical temperature profiles obtained at different instances of time. It clearly shows that the dominant thermal stratification is confined mainly to the ullage region. At the start of the simulation the temperature is almost constant in the liquid phase and then becomes slightly stratified a later times. In the ullage, the opposite effect, but a larger scale, takes place. It shows more extensive destratification that progresses monotonically with time.

\section{Conclusions}

The modeling of a partially filled sloshing cryostat tank undergoing thermal destratification was investigated. The CFD simulations incorporated models for inertial shaking and mass transfer at the interface. The system pressure decayed quickly and at a significant level in both the computational and experimental cases. The turbulent computational data with an accommodation coefficient of 0.01 produced the closest fit to the CNES pressure data. The sloshing free surface was found to initially oscillate to a single mode and then transitioned into secondary and tangential (swirl) wave modes. The temperature field in the ullage was characterized by the propagation of an energetic thermal convective layer generated at the sloshing free surface into the upper regions of the ullage. The temperature data also showed that sloshing free surface induced two distinct spatial and temporal thermal convective scales and periods of intermittency and periodic behavior before reaching an almost equilibrium state. At the end of 40 seconds the thermal stratification is significantly altered with maximum temperature ratio in the ullage reduced to about $80 \%$ of the initial normalized temperature.

\section{Acknowledgements}

The authors thank Olga Kartuzova, Mohammad Kassime, and Mark Stewart for their helpful suggestions and comments during the computational development. This work was conducted under an "Implementing Arrangement between the National Aeronautics and Space Administration of the United States of America and the Centre National D'Etudes Spatiales of France on Cooperation Related to Benchmarking Activities Regarding Computational Propellant Management Capability." The authors would like to thank Benjamin Legrand, Vincent Leudiere, and Pascal Fortunier of CNES. Without their help and discussions, this valuable cooperative activity would not have been possible.

This work was supported by the NASA Space Technology Mission Directorate's Technology Demonstration Missions Program under the Evolvable Cryogenics Project. 

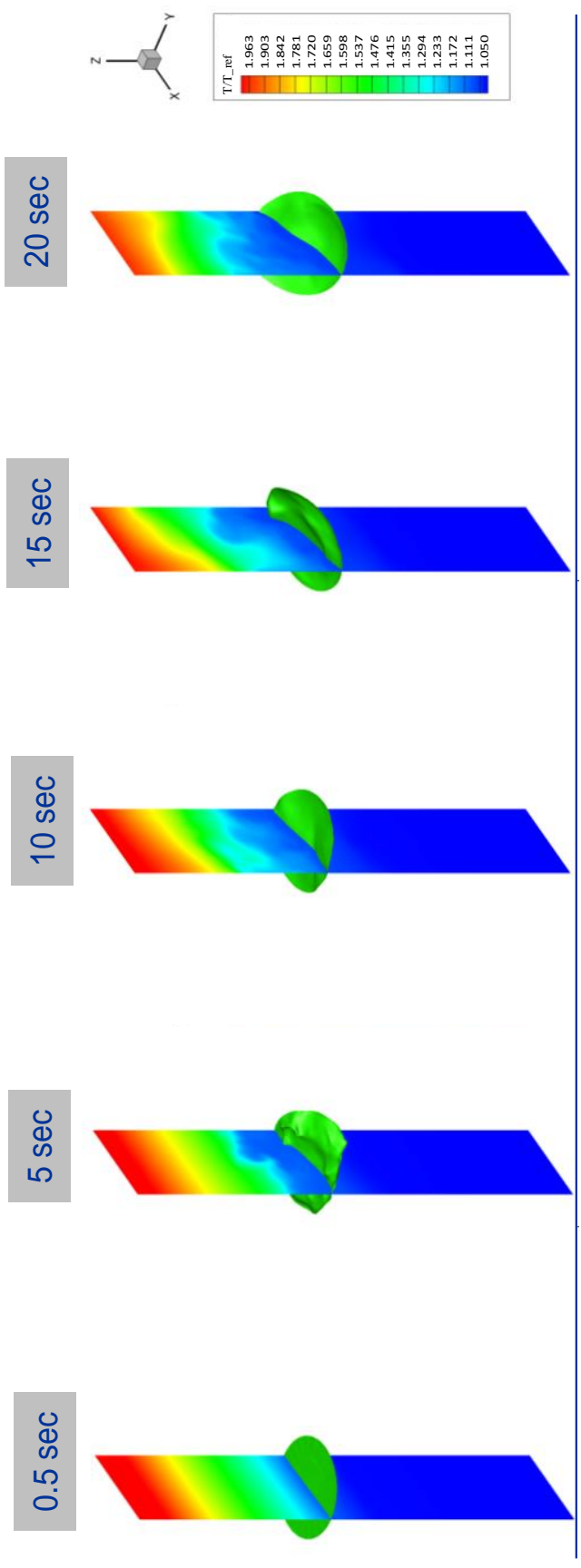

Figure 7: Time evolving temperature contours on the mid plane of the tank.

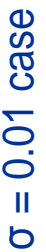
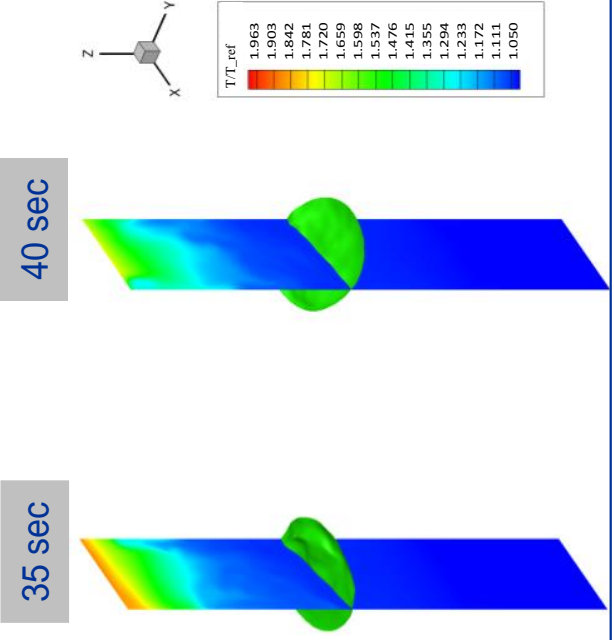

o

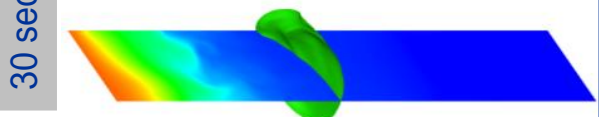

क

กิ

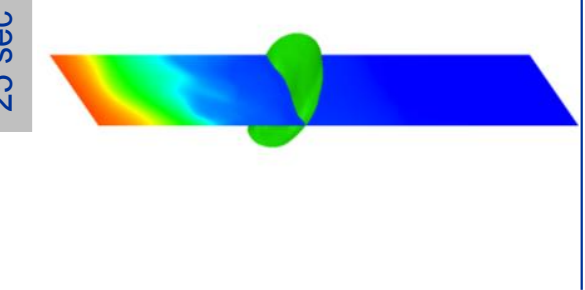




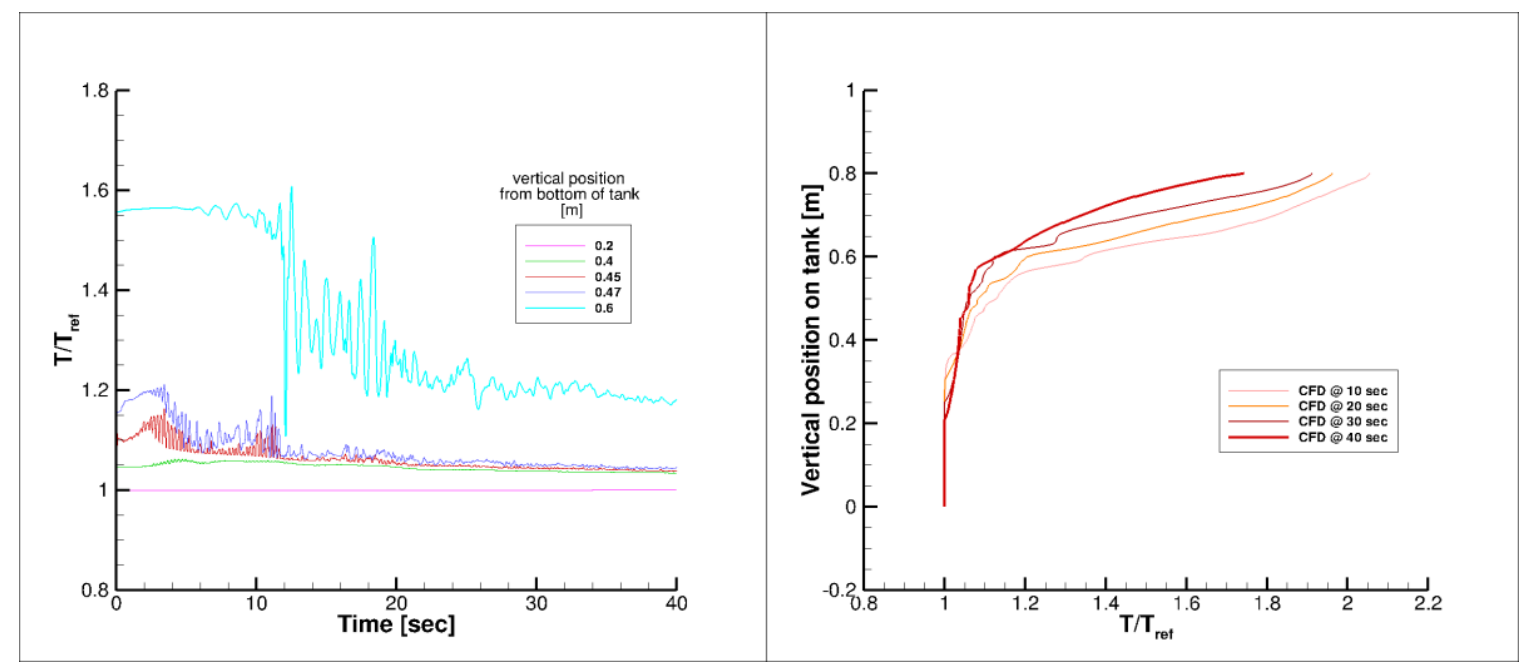

(a)

(b)

Figure 8: Temperature plots: (a) time history (b) vertical temperature profiles.

\section{References}

${ }^{1}$ Vatistas, G. H., Lin, S., and Kwok, C. K., "Reverse Flow Radius in Vortex Chambers," AIAA Journal, Vol. 24, No. 11, 1986, pp. $1872,1873$.

${ }^{1}$ Abramson, H. N. (1966) "The dynamic behavior of liquids in moving containers" NASA SP 106

${ }^{2}$ Dodge, F.T. (1991) "Analysis of small amplitude low gravity sloshing in axisymmetric tanks" Microgravity Sci. Technology. 4:228-34

${ }^{3}$ Abramson, H.N., Chu, W.-H. and Ransleben, G.E., Jr. "Representation of fuel sloshing in cylindrical tanks by an equivalent mechanical model," TR 8, Contract DA-23-072-ORD-1251, SwRI Project 43-768-2, Southwest Research Institute (June 1960); also ARS J. 31, 1697-1704 (1961).

${ }^{4}$ Lacapere, J., Vielle, B., and Legrand, B. (2009) "Experimental and Numerical Results of Sloshing with Cryogenic Fluids.” Progress in Propulsion Physics, vol. 1, pp. 267-278.

${ }^{5}$ Arndt, T., Dreyer, M., Behruzi, P., Winter, M., van Foreest, A. (2009) "Cryogenic Sloshing Tests in a Pressurized Cylindrical Reservoir." AIAA-2009-4860.

${ }^{6}$ Himeno, T., Sugimori, D., Ishikawa, K., Umemura, Y., Uzawa, S., Inoue, C., Watanabe, T., Nonaka, S., Nauruo, Y. and Inatani, Y. (2011) "Heat Exchange and Pressure Drop Enhanced by Sloshing." AIAA-2011-5682.

${ }^{7}$ J. U. Brackbill, D. B. Kothe, and C. Zemach. "A Continuum Method for Modeling Surface Tension". J. Comput. Phys.. 100. 335-354. 1992.

${ }^{8}$ Menter, F. R., "Two-Equation Eddy-Viscosity Turbulence Models for Engineering Applications,” AIAA Journal, Vol. 32 No. 8, 1994, pp. 1598-1605

${ }^{9}$ Schrage, R.W., A theoretical study of interphase mass transfer, Columbia University Press, New York, 1953.

American Institute of Aeronautics and Astronautics 